\title{
Proceeding
}

Supplementary Issue: Summer Conferences of Sports Science. Costa Blanca Sports Science Events, 25-26 September 2020. Alicante, Spain.

\section{Applying a menthol pain reliever prior to strength training reduces chronic low back pain and increases functional capacity in overweight or obese older adult}

NICOLE B. FRITZ1,2, JOAQUIN CALATAYUD ${ }^{1}$, PHIL PAGE ${ }^{3}$, MAHENDRA YADAV4, MOHAMMAD SIDIQ ${ }^{5}$, JUAN C. COLADO 6

${ }^{1}$ Research Unit in Sport and Health, University of Valencia, Valencia, Spain

2Health Department, University of Los Lagos, Puerto Montt, Chile

${ }^{3}$ Franciscan Missionary University of Baton Rouge, Louisiana, United States of America

${ }^{4}$ Department of Physiotherapy, Quality Care Medical Center, Abu Dhabi, United Arab Emirates

${ }^{5}$ Rehabilitation Medicine Department, Northern Area Armed Forces Hospital King Khalid, Military City, Saudi Arabia

${ }^{6}$ Department of Physical Education and Sports, University of Valencia, Valencia, Spain

\begin{abstract}
The combined effect of a muscle strength training program with the previous application of a topical menthol gel on the intensity of chronic low back pain (CLP) and the functional capacity of overweight/obese older women was evaluated. A randomized controlled clinical trial with parallel group comparison was conducted. In Phase 1 (12-weeks), 31 women were randomly assigned: (i) a control group that did not change their habits (CG; $n=11$ ); (ii) group of strength training prior to application of a gel with menthol $(\mathrm{RTM} ; n=10)$ and (iii) group that performed the same training prior to application of a placebo gel (RTP; $n=10)$. After 12 weeks of no training, phase 2 of the study (32-week duration) was run, where the RTM and RTP women were randomly reassigned. It was evaluated before and after each phase: body composition, functional capacity, pain perception and Oswestry disability index. The significance level established for the study was $p \leq .05$. Both training groups significantly improved all variables after stages 1 and 2 , with significant post-intervention differences but no significant intergroup differences, although RTM obtained better results. The percentage of improvement in pain perception and functional parameters were related to the intervention time without positive effects for CG. In conclusion, the use of menthol gel prior to a muscle strength program reduced pain and enhanced the functional improvements achieved as a result of moderate-high intensity training in older adult women with CLP and overweight or obesity.

Keywords: Spine; Musculoskeletal pain; Elastic bands; Topical gel; Body composition.

Cite this article as:

Fritz, N.B., Calatayud, J., Page, P., Yadav, M., Sidiq, M., \& Colado, J.C. (2020). Applying a menthol pain reliever prior to strength training reduces chronic low back pain and increases functional capacity in overweight or obese older adult. Journal of Human Sport and Exercise, 15(4proc), S1224-S1241. doi:https://doi.org/10.14198/ihse.2020.15.Proc4.24

Corresponding author. Department of Physical Education and Sports, University of Valencia. C/ Gascó Oliag 3. 46010, Valencia, Spain. https://orcid.org/0000-0002-3255-3940

E-mail: juan.colado@uv.es

Abstract submitted to: Spring Conferences of Sports Science. Costa Blanca Sports Science Events, 19-20 June 2020. Alicante, Spain.

JOURNAL OF HUMAN SPORT \& EXERCISE ISSN 1988-5202

(c) Faculty of Education. University of Alicante

doi:10.14198/jhse.2020.15.Proc4.24
\end{abstract}

S1224 $|2020|$ Proc4 | VOLUME 15

(C) 2020 University of Alicante 


\section{INTRODUCTION}

Spinal pain is the most prevalent type of musculoskeletal pain in older adults (OAs) (Edmond \& Felson, 2000; Podichetty et al., 2003), and it causes them to engage in less physical activities as it restricts or hinders their movements (Reid et al., 2005). The limitations produced by this type of pain worsen the usual decrease in motor performance or functional capacity promoted by the ageing process (Aagaard et al., 2010). Additionally, most patients with chronic low back pain (CLP) worsen their body composition because they fear such pain, and, as a result, they restrict their movements and generally refrain from engaging in any type of physical activity (Leboeuf-Yde et al., 2011). For these reasons, obesity and low muscle mass have been associated with the diagnosis of pain (Wright et al., 2010), and it has been reported that the prevalence of joint pain increases directly with higher values of body fat, especially when it comes from the lower part of the backbone (Vincent et al., 2011). At the same time, this worsening of body function and composition is associated simply or in combination with a decrease in life quality and life expectancy (Cedrashi et al., 2016; Vincent et al., 2011; Wright et al., 2010). Consequently, the evidence reported by previous research indicates that there is a large number of treatments for CLP because it is a serious problem for global public health (Katz, 2006). However, no single approach has been placed above the rest of the treatments since it has not yet been possible to determine which strategy is optimal (Haldeman \& Dagenais, 2008; Henchoz \& Kai-Lik, 2008). This situation makes it very difficult for doctors, physiotherapists, physical trainers, politicians, insurers, and the patients themselves to make decisions regarding which treatment or intervention is the most appropriate for the treatment of CLP and even more so in OAs (Searle et al., 2015).

Among different intervention strategies that are present (i.e., surgery, pharmacological treatment, and nonmedical interventions) (Searle et al., 2015; van Middelkoop et al., 2010), treatment with physical exercise is probably the type of conservative treatment most used worldwide, whose efficacy has been demonstrated by improving all the health alterations previously mentioned in isolation: pain, loss of function, and worsening of body composition (Foster et al., 2016; Hubley-Kozey, 2003).

Because reduced strength (Hubley-Kozey, 2003), flexibility (Vincent et al., 2011), and aerobic endurance (van der Velde \& Mierau, 2000) are present in many people with CLP, exercise programs to treat CLP generally include training for these physical qualities (Foster et al., 2016; van Middelkoop et al., 2010; van Tulder et al., 2000). Among these indicated physical exercise protocols, those that focus on the improvement of muscular strength currently stand out given the great benefits they provide for various population types when carried out with the use of various device types (i.e. own body weight, free weight, machines, elastic bands, aquatic environment, etc.) (Hayden et al., 2005; Searle et al., 2015). However, there is still no clear recommendation regarding the prescription of this type of physical exercise based on muscle strength training in OAs for joint improvement of both spinal pain and physical function and body composition, although it is widely recognized that such an intervention could be effective (Foster et al., 2018; Ishak et al., 2016).

Among the other alternative treatments to exercise mentioned, it should be noted that there are currently topical gels that are used for analgesic purposes to relieve discomfort and pain in the locomotor system. Among these, the gels that contain the active ingredient of menthol are highlighted since they do not present collateral adverse effects usual in other types of local analgesics (Sundstrup et al., 2014). The positive effects of this type of gel, which is recommended to be applied in various dosages throughout the day, have been corroborated acutely and chronically (Sundstrup et al., 2014; Zhang et al., 2008). Some scientific studies have associated its use with physical activities, both just after training in subjects without musculoskeletal pain to minimize the loss of physical performance and muscle pain typical of intensive physical exercise programs (Johar et al., 2012), and just before performing different functional tasks in subjects with knee pain 
to reduce pain and increase performance during the activity (Topp et al., 2013). A possible justification for the complementary use of menthol together with physical exercise is based on the fact that menthol activates TRP-M8 receptors, generating a sensation of cold in sensory neurons of the skin and causing an effect similar to that of cryotherapy with the important difference that it does not alter muscle strength after its application (Page \& Alexander, 2017) and maintains a half-life of 96 minutes after its transdermal application (Martin et al., 2004), which indicates that it can be used during a training session.

Due to all these pieces of evidence explained up to here, it is possible to wonder if the application of a topical gel with menthol prior to a muscle strengthening session can reduce the usual muscle inhibition due to pain and allow training without restricting movements and in a controlled manner at an intensity that in the medium and long term can cause decreases in low back pain and improvements in physical functions and body composition in OAs. It was hypothesized that the application of the menthol gel prior to the resistance training program would generate greater benefits than the application of physical training alone in reducing pain and improving body function and composition in the short and long term.

\section{METHODS}

\section{An experimental approach to the problem}

A randomized controlled clinical trial, with a comparison of parallel groups and allocation ratio of 1:1. The study was approved by the ethics committee of the University of Valencia (H1475430627434). All participants signed an informed consent form, and the research was carried out in accordance with the Declaration of Helsinki (2008 revision). The study adhered to the CONSORT Statement to ensure transparent and standardized information. The study was divided into two phases (Figure 1).

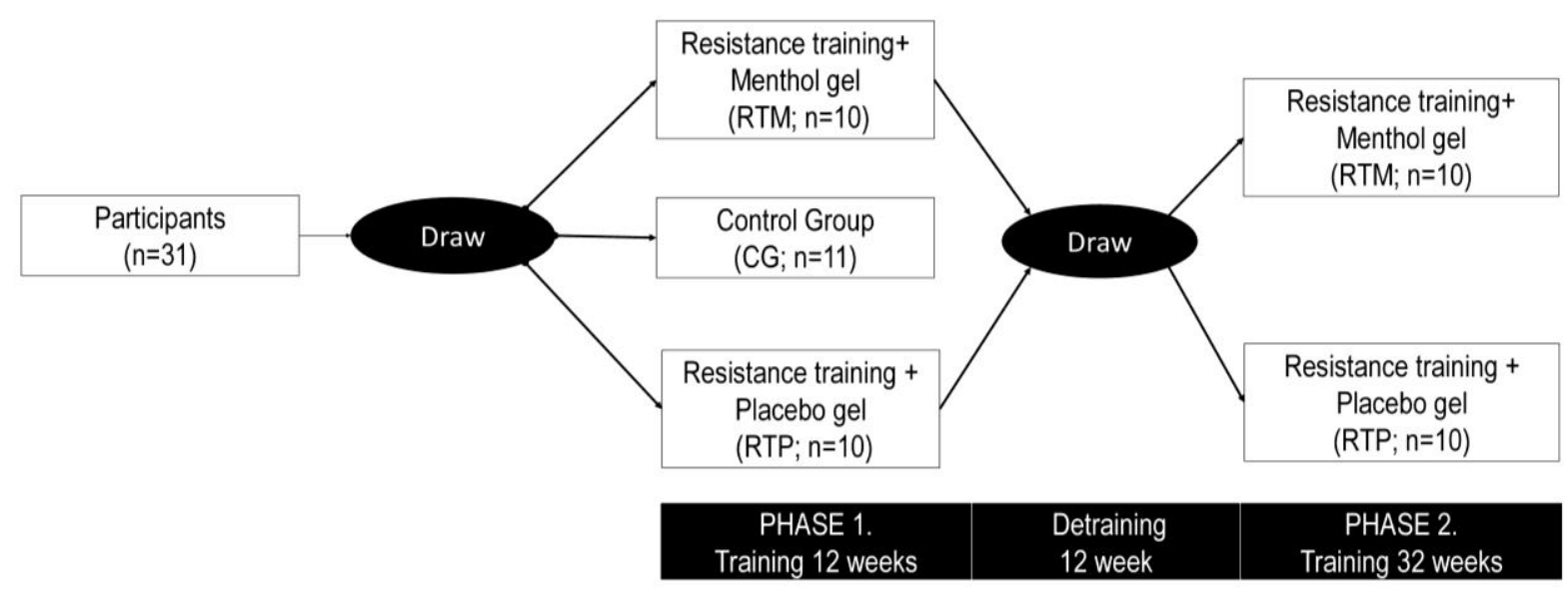

Figure 1. Study design.

- $\quad$ Phase 1: Thirty-one women were randomly assigned to three groups: a training group with elastic resistance prior to the application of the menthol gel (RTM; $n=10)$, a second training group with elastic resistance prior to the application of a placebo gel (RTP; $n=10)$, and a control group (CG; $n$ $=11$ ) that did not undergo training.

- Phase 2: After 12 weeks of detraining, with the aim of comparing the short and long-term effects of the menthol gel, the 20 OAs who participated in phase 1 were again randomized into two groups: $\operatorname{RTM}(n=10)$ and $\operatorname{RTP}(n=10)$ groups. 


\section{Participants}

OAs from the Valdivian community (Chile) with a history of unspecific CLP were invited to participate through a call in the local press and the National Older Adults Service (SENAMA Los Ríos, Chile). For the selection of the sample, the following inclusion criteria were taken into account: age $\geq 60$ years, BMl $\geq 25 \mathrm{~kg} / \mathrm{m}^{2}$, presence of non-specific low back pain without peripheral radiation, pain $>3$ months, medical authorization to perform physical activities, and being able to sign an informed consent form. Exclusion criteria were the presence of a neurodegenerative or inflammatory disease in the spine, a lumbar disc herniation that requires surgical treatment, vertebral fractures, history of lumbar surgery, severe neoplastic, psychiatric or cardiopulmonary disease, neuromuscular, sensory, or cognitive alterations that prevented the practice of exercise, the use of physiotherapeutic treatment for the spine in the last three months, the practice of physical exercise during study and fluctuation of body weight greater than $2 \mathrm{~kg}$ during the previous year.

\section{Sample}

The calculation of the sample size was carried out based on the values previously published in the literature by Hägg et al. (2003) and Ostelo \& de Vet (2005) for the visual analogue scale pain (VAS), in which a minimum difference of $20 \mathrm{~mm}$ is described at a $95 \%$ tolerance interval for the finding to be considered clinically important, using the free software GRANMO (version 7.12, April 2012).

\section{Procedures}

In the previous week, week 12 and 32 the evaluations were carried out, each one on three different days. The evaluators were three physical therapists with five years of experience in OA care. On the first day, weight, body mass index (BMI), fat and lean mass were evaluated using bioelectrical impedance (Tanita ${ }^{\circledR}$ TFS300GS), and waist and hip circumference was assessed following the NAHNES standards (2009). During all evaluations, the participants were barefoot and wore light clothing. On the second day, functional ability tests were assessed:

- 6-minute walking test (6MWT): the participants were asked to walk as fast as possible, without running, for six minutes on a flat surface marked $30 \mathrm{~m}$. The internal consistency values of the test are 0.90-0.96 (Rikli \& Jones, 2013).

- $\quad$ Timed-up-and-go (TUG): the participants were asked to get up in one go and walk and turn around the cone located at three meters and sit down again. TUG has excellent intra-ratter reliability (ICC = 0.94) in the OA community (Hofheinz \& Schusterschitz, 2010).

- Single-leg-stance: the participants crossed their arms on the thorax, letting the hands rest on the shoulders, and performed triple-flexion of one leg at $90^{\circ}$. They held the position for as long as possible on one foot. The coefficient of the intra-class correlation of the test is 0.994 (Springer et al., 2007).

- Chair-stance: the participants were asked to rise to a full standing position from a chair and sit back down again as many times as possible during $30 \mathrm{~s}$. It was performed only once and has an internal consistency of 0.79-0.93 (Rikli \& Jones, 2013).

- Grip-strength: a Jamar® hydraulic dynamometer (PC 5030 J1, Sammons Preston Rolyan, USA) was used. From the sitting position with the arm adducted, the elbow flexed at $90^{\circ}$, and the wrist in a neutral position, the participants were asked to hold the dynamometer in position II while the evaluator supported it slightly from the base (Boscheinen-Morrin \& Conolly, 2001). Due to its predictive validity and simplicity, this test is considered a useful vital sign for the functional assessment of OAs.

- Maximum isometric force of upper and lower limbs: the Upright rowing (UR) and Squatting (SQ) tests were applied according to the protocol described by Colado et al. (2010). The force-time curve of the 
peak traction was captured by a load cell and the analysis of the force signal was performed using the MATLAB 7.0 program (MathWorks Inc., Natick, MA, USA).

- Curl-up test: assesses abdominal resistance, by maintaining the trunk flexion without the lower pole of the scapulae touching the table while keeping the knees bent $90^{\circ}$ with the arms crossed at the chest level and feet under a strap at the ankles. The best time of two attempts after 1 min rest was recorded (Chen et al., 2009).

On the third day, self-perceived functional health tests were applied by assessing pain with the visual analogue scale (VAS), where the participating OAs marked on a $100 \mathrm{~mm}$ line the point that best described the intensity of pain, and the questionnaire for the Oswestry Disability Index (ODI), which evaluates the alternative that best represents their current condition for 10 activities of daily living (Fairbank \& Pynsent, 2000).

\section{Intervention plan}

The plan was carried out in an OA meeting house. The participants were familiarized with the first four sessions so that they could be instructed on the correct execution of the exercises, the regulation of intensity with elastic bands, and the control of breathing. Subsequently, the groups carried out a training program periodized in 12 weeks for phase 1 and 32 weeks for phase 2. These periods were preferred because the literature mentions that resistance training causes hypertrophy in a minimum period of 8 to 12 weeks, which was considered a "short term" for this work, while in a longer training period, these effects are maintained (32 weeks = "long term") (Steib et al., 2010). The workouts had a frequency of two weekly sessions on alternate days with a 48-hour rest between sessions. The large muscle groups of the upper and lower limb and trunk were trained, following the previously published methodology for this type of population (Fritz et al., 2018; Gargallo et al., 2019). The person in charge of the centre was in charge of controlling the attendance and compliance with the intervention plan.

Intervention groups

Both groups trained together and in a professionally supervised manner. During the first six weeks, they trained with three sets of 10 repetitions for each exercise, maintaining a range of local perceived effort of 6 7, according to the ratio established on the Rating of Perceived Exertion (RPE) for training with elastic bands in OAs (Colado et al., 2018) and with a 90 s active pause between sets. In the following six weeks, the RPE was increased to 8-9, the training was increased to four sets per exercise, maintaining an active rest of $90 \mathrm{~s}$ between sets until week 8 . The duration of active rest was later reduced to $60 \mathrm{~s}$ during the last four weeks. For phase 2, the same prescription was maintained and in the last 12 weeks, the duration of active rest was reduced to $45 \mathrm{~s}$. All sessions lasted $60 \mathrm{~min}$, which was divided into warm-up (10 min), training (40 min), and cool down-stretching (10 min). Elastic bands (CLX Theraband $\mathrm{B}, \mathrm{Akron}, \mathrm{OH}, \mathrm{USA}$ ) of different strengths (blue, black, silver, and gold) were used to carry out the strength exercises.

The menthol gel used in the study was Biofreeze® (TheraBand®; Hygenic Corporation, Akron, OH, USA), and the placebo gel was supplied by the same company. The placebo gel had the same composition as the normal gel, but without the menthol. The roll-on gel is easy to self-apply by this type of population. Always supervised by the trainer, the participants were instructed to self-apply the menthol gel just 10 min before the start of the training session. To do this, the exercisers cleaned their lumbar area with a damp towel to remove residues from the skin and then carried out the application with the roll-on in the following way indicated by the manufacturer: two vertical applications right and left and two horizontal applications claimed to cover the lumbar area from T12 to L5. Consecutive self-applications of the gel were carried out by each participant, 
and the application instructions previously given were reinforced by means of a poster displayed at the training site. This action was replicated, from week 1 of training to phase 2 of the study.

Control group (CG)

They were instructed not to modify their usual daily activities, not to carry out any physical activities, and to report any change in diet or the start of physical or medical treatment. With the supervision of the investigators, the person in charge of the centre made telephone contact once a week to verify these conditions and to inform whether the participant was in the city.

\section{Randomization and blinding}

The participants, each of whom was assigned a code, were randomized to experimental groups (RTM and RTP groups) or the control group (CG) (two groups of 12 people and one of 11). Regarding the use of the topical gel, the person responsible for developing the training sessions received it blindly in the form of a rollon labelled with a reference code applied by the supplier. The placebo gel had the same colour, scent, and texture as that containing menthol. In addition, each roll-on, bearing the name of the participant using it, was placed by the trainer so that the participants always used the same roll-on in each training session. According to the code previously assigned to each roll-on and without knowledge of the results at the end of the study, the supplier revealed to the researchers which code of each roll-on belonged to the menthol gel or the placebo gel.

\section{Statistical analyses}

The normality of the data was confirmed with the Kolmogorov-Smirnov test. To compare the participants in the experimental groups and the control group, both of whom were measured at the beginning and the end of the intervention program, and to determine if there were any changes over time, the analysis of variance ANOVA (SPSSv23., SPSS, Inc., Chicago, Illinois, USA) was used. When significant differences were found on some of the dependent variables, a Bonferroni post hoc analysis was performed to determine the source of the difference. To determine if the statistically significant difference was of practical interest, the effect sizes (Cohen's) and the intragroup percentage change were calculated for each of the phases and intergroup percentage changes were calculated for phase 2 . The results were interpreted following the standards established by Cohen where: $<0.2$ is considered a trivial effect; $0.2-0.5$ is indicative of a small effect; 0.5 0.8 moderate effect and $>0.8$ a large effect size. For the tests used, a value of $p \leq .05$ was assumed as evidence of a statistically significant finding.

\section{RESULTS}

The size of the sample began with 53 potential OAs, of whom 35 met the inclusion criteria, only women agreeing to participate. The average age was $71.13 \pm 6.47$ years (MIN: 62; MAX: 85 years), reporting "moderate" back pain according to VAS and a percentage of disability of "moderate to severe" according to the ODI classification. The flow of participants is detailed in Figure 2.

The main exclusion criterion was the presence of surgical resolution neurodegenerative pathology in the spine. None of the participants in the intervention group dropped out of the study; they were only excluded due to low attendance due to medical causes not derived from the intervention. In the second phase of the study, which lasted 32 weeks, the same participants who completed phase 1 were retained, while the CG was not considered, because it started recreational dance workshops organized by the institution. 
The attendance rate for phase 1 of the program was $91.7 \%$, while for phase 2 it was $89.3 \%$. The baseline characteristics of the participants are presented in Table 1. It should be noted that throughout the study, none of the participants reported harm, worsening of their baseline health condition, or injury due to the intervention program.

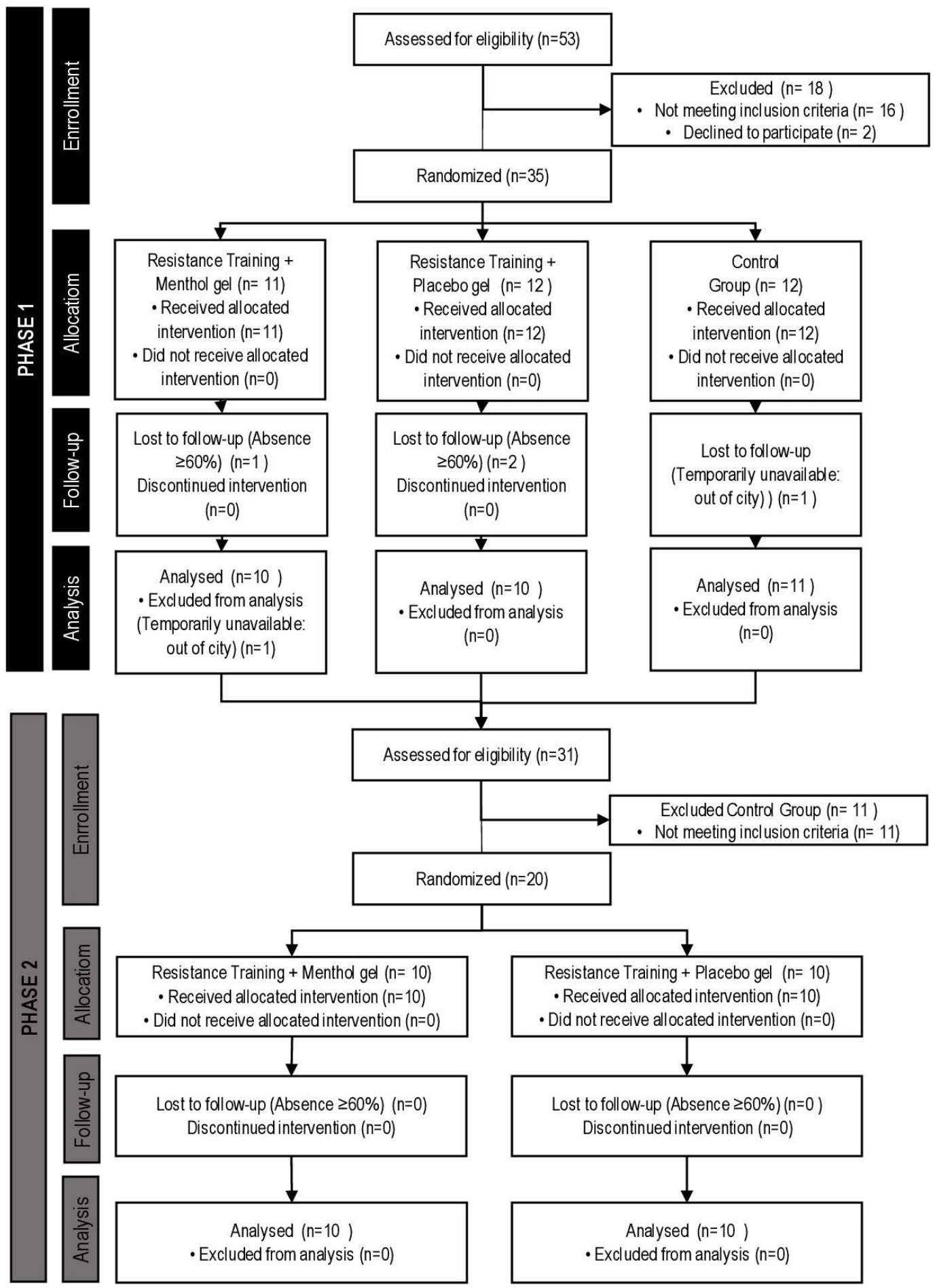

Figure 2. Consort flow diagram. 
Table 1. Baseline characteristic.

\begin{tabular}{llll}
\hline Characteristic & RTM $(\mathbf{n}=\mathbf{1 0})$ & RTP $(\mathbf{n}=\mathbf{1 0})$ & CG $(\mathbf{n}=11)$ \\
\hline Age $($ years $)$ & $70.3 \pm 6.3$ & $71.0 \pm 6.9$ & $72.0 \pm 6.9$ \\
Weight $(\mathrm{kg})$ & $67.8 \pm 9.4$ & $74.5 \pm 11.6$ & $70.6 \pm 11.9$ \\
Height $(\mathrm{m})$ & $1.50 \pm 0.1$ & $1.52 \pm 0.1$ & $1.50 \pm 0.1$ \\
BMI $(\mathrm{kg} / \mathrm{m} 2)$ & $30.1 \pm 3.5$ & $32.4 \pm 4.2$ & $30.4 \pm 4.1$ \\
VAS $\left(\mathrm{n}^{\circ}\right)$ & $6.5 \pm 1.4$ & $6.4 \pm 2.0$ & $5.9 \pm 1.5$ \\
ODI $(\%)$ & $41.8 \pm 10.6$ & $39.6 \pm 12.1$ & $38.2 \pm 14.8$ \\
\hline
\end{tabular}

Note. Data are presented as the Mean \pm Standard Deviation. Abbreviations: BMl= Body Mass Index; RTM = resistance training group +menthol gel; $R T P=$ resistance training group + placebo gel; $C G=$ control group.

\section{Effect of phase 1 intervention: initial 12 weeks}

The changes in body composition and functional health in phase 1 are presented in Table 2. Regarding the body composition variables, the RTM and RTP training groups improved all variables with a small effect size $(E S=0.2-0.5)$, however, the RTM group was the one that obtained the greatest significant intragroup changes $(p \leq .01)$ for all variables except for lean mass. There were no significant differences between the groups.

Regarding the functional health variables, the RTM and RTP training groups improved all variables without differences between groups. The RTM group was the one that presented greater improvements compared to the RTP group in all variables with significant intragroup differences ( $p \leq .01)$, except for the curl test and high effect size for the changes in the variables: 6MWT, TUG, Chair-stance, VAS, and ODI. The RTP group improved all its functional health variables, with these intragroup improvements being significant for all variables, except for TUG and squatting. On the other hand, the CG obtained either a decrease or no significant difference in all variables of body composition and functional health, with a high effect size (ES > $0.8)$. The VAS and ODI variables of both training groups were significantly lower post-training.

\section{Effects of phase 2 intervention: 32 weeks}

The changes in body composition and functional health of phase 2 are presented in Tables 3 and 4 . Both groups significantly improved all body composition variables without significant intergroup differences at week 12 and 32, obtaining greater results as training time increased, with a small effect size (ES: $0.2-0.5)$ both within and between groups. However, it was the RTM group that obtained greater improvements compared to the group that trained with placebo. The variable that presented the greatest improvement was the fat mass $(12.5 \%)$ after 32 weeks of intervention for the group that trained with menthol. Regarding the functional health variables, all the parameters improved as the intervention time increased, with the greatest improvements being for the menthol group, although without significant differences between intervention groups.

The variables that presented the greatest percentage changes after 32 weeks of training were the balance variables: TUG and Single-leg-stance, with a high effect size for both groups. Finally, the variables that evaluated low back pain (VAS) and percentage of disability (ODI) showed a percentage of significant improvements greater than $60 \%$ and high effect size for both groups, without significant long-term intergroup differences. 
Table 2. Intervention effects on corporal composition and functional health for Phase 1 (12 weeks).

\begin{tabular}{|c|c|c|c|c|c|c|c|c|c|c|c|c|}
\hline & \multicolumn{4}{|c|}{$\operatorname{RTM}(n=10)$} & \multicolumn{4}{|c|}{$\operatorname{RTP}(n=11)$} & \multicolumn{4}{|c|}{ CG $(n=10)$} \\
\hline & $\begin{array}{l}\text { Pretest } \\
\text { SD }\end{array}$ & $\begin{array}{l}\text { Postest } \\
\text { SD }\end{array}$ & $\% \Delta$ & $\begin{array}{l}\text { ES } \\
\text { (d) }\end{array}$ & $\begin{array}{l}\text { Pretest } \\
\text { SD }\end{array}$ & $\begin{array}{c}\text { Postest } \\
\text { SD }\end{array}$ & $\% \Delta$ & $\begin{array}{l}\text { ES } \\
\text { (d) }\end{array}$ & $\begin{array}{l}\text { Pretest } \\
\text { SD }\end{array}$ & $\begin{array}{l}\text { Postest } \\
\text { SD }\end{array}$ & $\% \Delta$ & $\begin{array}{l}\text { ES } \\
\text { (d) }\end{array}$ \\
\hline \multicolumn{13}{|l|}{ Corporal Composition } \\
\hline Weight $(\mathrm{kg})$ & $67.8 \pm 9.4$ & $66.4 \pm 9.3$ & $-2.1^{* *}$ & -0.2 & $74.4 \pm 11.6$ & $73.8 \pm 11.5$ & -0.9 & -0.1 & $70.6 \pm 11.9$ & $70.8 \pm 12.1$ & +0.2 & 0.0 \\
\hline $\mathrm{BMI}\left(\mathrm{kg} / \mathrm{m}^{2}\right)$ & $30.1 \pm 3.5$ & $29.4 \pm 3.4$ & $-2.2^{* *}$ & -0.2 & $32.4 \pm 4.2$ & $32.1 \pm 4.0$ & -0.9 & -0.1 & $30.4 \pm 4.1$ & $30.5 \pm 4.2$ & +0.2 & 0.0 \\
\hline Fat mass $(\mathrm{kg})$ & $27.8 \pm 6.6$ & $26.3 \pm 6.3$ & $-5.2^{* *}$ & -0.2 & $33.5 \pm 6.4$ & $32.8 \pm 6.0$ & -2.3 & -0.1 & $29.8 \pm 6.7$ & $30.1 \pm 6.9$ & +0.9 & 0.0 \\
\hline Lean mass $(\mathrm{kg})$ & $39.6 \pm 3.4$ & $40.8 \pm 3.8$ & +3.4 & 0.3 & $41.3 \pm 4.9$ & $42.3 \pm 5.1$ & +2.5 & 0.2 & $41.4 \pm 4.9$ & $41.0 \pm 4.8$ & -0.9 & -0.1 \\
\hline Waist-circumference $(\mathrm{cm})$ & $97.4 \pm 7.6$ & $94.8 \pm 7.8$ & $-2.7^{* *}$ & -0.3 & $101.7 \pm 8.3$ & $98.3 \pm 8.7$ & $-3.3^{\text {** }}$ & -0.4 & $99.8 \pm 9.7$ & $98.8 \pm 11.2$ & -1 & -0.1 \\
\hline Hip-circumfernce (cm) & $107.1 \pm 6.2$ & $104.6 \pm 5.3$ & $-2.3^{* *}$ & -0.4 & $110.6 \pm 6.6$ & $109.2 \pm 6.2$ & -1.3 & -0.2 & $108.8 \pm 7.6$ & $109.4 \pm 8.1$ & +0.5 & -0.1 \\
\hline \multicolumn{13}{|l|}{ Functional Health } \\
\hline $6 \mathrm{MWT}(\mathrm{m})$ & $400.6 \pm 69.8$ & $484.1 \pm 50.8$ & $+20.8^{* *}$ & -1.4 & $399.9 \pm 92.0$ & $453.5 \pm 74.9$ & $+13.4^{* *}$ & -0.6 & $426.0 \pm 77.0$ & $420.3 \pm 77.7$ & -1.4 & 0.1 \\
\hline TUG (s) & $6.8 \pm 1.6$ & $4.7 \pm 0.4$ & $-30.1^{* *}$ & 1.8 & $7.1 \pm 0.9$ & $5.5 \pm 0.9$ & $-22.2^{* *}$ & 1.7 & $6.7 \pm 0.9$ & $6.8 \pm 1.1$ & $+1.1 \S$ & -0.1 \\
\hline Single-leg-stance (s) & $10.9 \pm 11.0$ & $18.3 \pm 14.7$ & $+68.5^{* *}$ & -0.6 & $8.9 \pm 10.1$ & $12.7 \pm 10.9$ & +41.3 & -0.4 & $11.7 \pm 16.9$ & $10.9 \pm 17.1$ & -6.9 & 0.0 \\
\hline Chair-stance (n) & $15.1 \pm 2.1$ & $19.0 \pm 3.0$ & $+25.8^{* *}$ & -1.5 & $14.7 \pm 5.8$ & $16.9 \pm 6.5$ & $+14.9^{* *}$ & -0.4 & $14.8 \pm 4.6$ & $14.3 \pm 5.1$ & -3.7 & 0.1 \\
\hline Grip-strength (kg) & $22.6 \pm 4.2$ & $25.4 \pm 4.8$ & $+12.4^{* *}$ & -0.6 & $23.1 \pm 4.5$ & $25.6 \pm 5.2$ & $+10.8^{* *}$ & -0.5 & $23.3 \pm 4.8$ & $22.7 \pm 4.7$ & -2.3 & 0.1 \\
\hline Upringth-rowing (kg) & $24.1 \pm 7.1$ & $29.0 \pm 8.2$ & $+20.3^{* *}$ & -0.6 & $24.7 \pm 7.9$ & $27.7 \pm 6.4$ & $+12.5^{* *}$ & -0.4 & $26.0 \pm 6.8$ & $23.7 \pm 6.1$ & -8.8 & 0.4 \\
\hline Squatting (kg) & $31.4 \pm 12.1$ & $41.0 \pm 15.5$ & $+30.7^{* *}$ & -0.7 & $34.6 \pm 12.4$ & $39.2 \pm 13.1$ & +13.1 & -0.4 & $29.0 \pm 10.7$ & $27.7 \pm 10.1$ & -4.5 & 0.1 \\
\hline Curl-up (s) & $6.7 \pm 11.8$ & $9.6 \pm 12.6$ & +44.3 & -0.2 & $17.9 \pm 28.5$ & $24.1 \pm 30.1$ & $+34.2^{*}$ & -0.2 & $8.5 \pm 10.5$ & $7.1 \pm 9.3$ & -16.6 & 0.1 \\
\hline $\operatorname{VAS}\left(n^{\circ}\right)$ & $6.5 \pm 1.4$ & $0.3 \pm 0.7$ & $-95.4^{* *}$ & 5.6 & $6.4 \pm 2.0$ & $0.4 \pm 0.9$ & $-93.8^{* *}$ & 3.8 & $5.9 \pm 1.5$ & $5.5 \pm 2.2$ & $-6.2 \S$ & 0.2 \\
\hline ODI (\%) & $41.8 \pm 10.6$ & $17.4 \pm 11.4$ & $-58.4^{* *}$ & 2.2 & $39.6 \pm 12.1$ & $18 \pm 4.2$ & $-54.6^{* *}$ & 1.6 & $38.2 \pm 14.8$ & $41.8 \pm 13.9$ & $+9.5 \S$ & -0.3 \\
\hline
\end{tabular}

Note. Data are presented as the Mean \pm Standard Deviation. Abbreviations: $R T M=$ Resistance Training Group + menthol gel; RTP = resistance training group + placebo gel; $C G=$ Control Group; $\Delta \%$ = percentage change from pre- to postest; ES: Effect Size; BMI: Body Mass Index; 6MWT: 6minutes walks test; TUG: timed up and go; VAS: Visual Analogue Scale; ODI: Oswestry disability index. ES: $<0.2=$ trivial effect; $0.2-0.5=$ small effect; $0.5-0.8=$ moderate effect and $>0.8=$ large effect; Intragroup differences $\left({ }^{*} p \leq .05\right.$ and ${ }^{* *} p \leq$ .01); § Intergroup difference CG vs BGE and PGE ( $p \leq .01)$. 
Table 3. Intervention effects on corporal composition for Phase 2 (32 weeks).

\begin{tabular}{|c|c|c|c|c|c|c|c|c|c|c|c|c|}
\hline & \multirow{2}{*}{ Group } & \multirow{2}{*}{$\begin{array}{c}\text { Pretest } \\
\text { (Baseline) }\end{array}$} & \multirow{2}{*}{$\begin{array}{c}\text { Inte-test } \\
\text { (Week 12) }\end{array}$} & \multirow{2}{*}{$\begin{array}{c}\text { Postest } \\
\text { (Week 32) }\end{array}$} & \multicolumn{3}{|c|}{ Pre-intertest } & \multicolumn{3}{|c|}{ Pre-postest } & \multicolumn{2}{|c|}{ ES (d) intergroup } \\
\hline & & & & & $\Delta \%$ & $\mathrm{ES}(\mathrm{d})$ & $p$ value & $\Delta \%$ & ES (d) & $p$ value & Intertest & Postest \\
\hline \multirow{2}{*}{ Weight (kg) } & RTM & $66.8 \pm 11.1$ & $65.6 \pm 11.2$ & $64.7 \pm 11.2$ & $-1.8^{* *}$ & -0.1 & .00 & $-3.1^{\text {t* }}$ & -0.2 & .00 & \multirow{2}{*}{0.2} & \multirow{2}{*}{0.2} \\
\hline & RTP & $67.7 \pm 9.9$ & $67.2 \pm 9.9$ & $66.8 \pm 10.0$ & $-0.7^{* *}$ & -0.1 & .00 & $-1.2^{* *}$ & -0.1 & .02 & & \\
\hline \multirow{2}{*}{$\mathrm{BMI}\left(\mathrm{kg} / \mathrm{m}^{2}\right)$} & RTM & $29.2 \pm 3.6$ & $28.7 \pm 3.7$ & $28.3 \pm 3.7$ & $-1.9^{* *}$ & -0.2 & .00 & $-3.2^{* *}$ & -0.3 & .00 & \multirow{2}{*}{0.3} & \multirow{2}{*}{0.4} \\
\hline & RTP & $30.2 \pm 3.8$ & $29.9 \pm 3.7$ & $29.8 \pm 3.6$ & $-0.7^{* *}$ & -0.1 & .00 & $-1.3^{* *}$ & -0.1 & .01 & & \\
\hline \multirow{2}{*}{ Fat mass $(\mathrm{kg})$} & RTM & $26.5 \pm 7.3$ & $24.3 \pm 6.8$ & $23.2 \pm 7.2$ & $-7.8^{* *}$ & -0.3 & .00 & $-12.5^{* *}$ & -0.5 & .00 & \multirow{2}{*}{0.4} & \multirow{2}{*}{0.5} \\
\hline & RTP & $27.4 \pm 6.3$ & $26.9 \pm 6.1$ & $26.2 \pm 5.6$ & -1.9 & -0.1 & .1 & $-4.6 *$ & -0.2 & .01 & & \\
\hline \multirow{2}{*}{ Lean mass (kg) } & RTM & $40.3 \pm 4.2$ & $41.1 \pm 4.9$ & $41.5 \pm 4.6$ & $+2.1^{*}$ & 0.2 & .04 & $+3.2^{* *}$ & 0.3 & .01 & \multirow{2}{*}{-0.2} & \multirow{2}{*}{-0.2} \\
\hline & RTP & $40.2 \pm 4.7$ & $40.3 \pm 4.9$ & $40.7 \pm 5.1$ & $+0.1^{*}$ & 0.0 & .02 & $+1.2^{*}$ & 0.0 & .02 & & \\
\hline \multirow{2}{*}{ Waist-circumference $(\mathrm{cm})$} & RTM & $95.6 \pm 11.9$ & $93.6 \pm 12.1$ & $92 \pm 11.9$ & $-2.1^{* *}$ & -0.2 & .00 & $-3.7^{* *}$ & -0.3 & .00 & \multirow{2}{*}{0.1} & \multirow{2}{*}{0.1} \\
\hline & RTP & $96.3 \pm 8.4$ & $94.6 \pm 8.5$ & $93.1 \pm 8.6$ & $-1.8^{* *}$ & -0.2 & .00 & $-3.3^{* *}$ & -0.3 & .00 & & \\
\hline \multirow{2}{*}{ Hip-circumfernce (cm) } & RTM & $102.4 \pm 6.9$ & $101.1 \pm 6.5$ & $100.6 \pm 6.3$ & $-1.3^{* *}$ & -0.2 & .01 & $-1.8^{* *}$ & -0.3 & .01 & \multirow{2}{*}{0.3} & \multirow{2}{*}{0.1} \\
\hline & RTP & $103.8 \pm 8.3$ & $103 \pm 7.9$ & $101.5 \pm 7.7$ & -0.8 & -0.1 & .12 & $-2.2^{* *}$ & -0.3 & .00 & & \\
\hline
\end{tabular}

Note. Data are presented as the Mean \pm Standard Deviation. Abbreviations: $R T M=$ resistance training group + menthol gel; RTP = Resistance Training Group + placebo gel; CG = Control Group; $\Delta \%$ = percentage change from pre- to postest; ES: Effect Size; BMI: Body Mass Index; 6MWT: 6minutes walks test; TUG: timed up and go; VAS: Visual Analogue Scale; ODI: Oswestry disability index. ES: $<0.2=$ trivial effect; $0.2-0.5=$ small effect; $0.5-0.8=$ moderate effect and $>0.8=$ large effect; Intragroup differences $\left({ }^{*} p \leq .05\right.$ and ${ }^{* *} p \leq$ .01); § Intergroup difference ( $p \leq .01)$. 
Table 4. Intervention effects on Functional Health for Phase 2 (12 and 32 weeks).

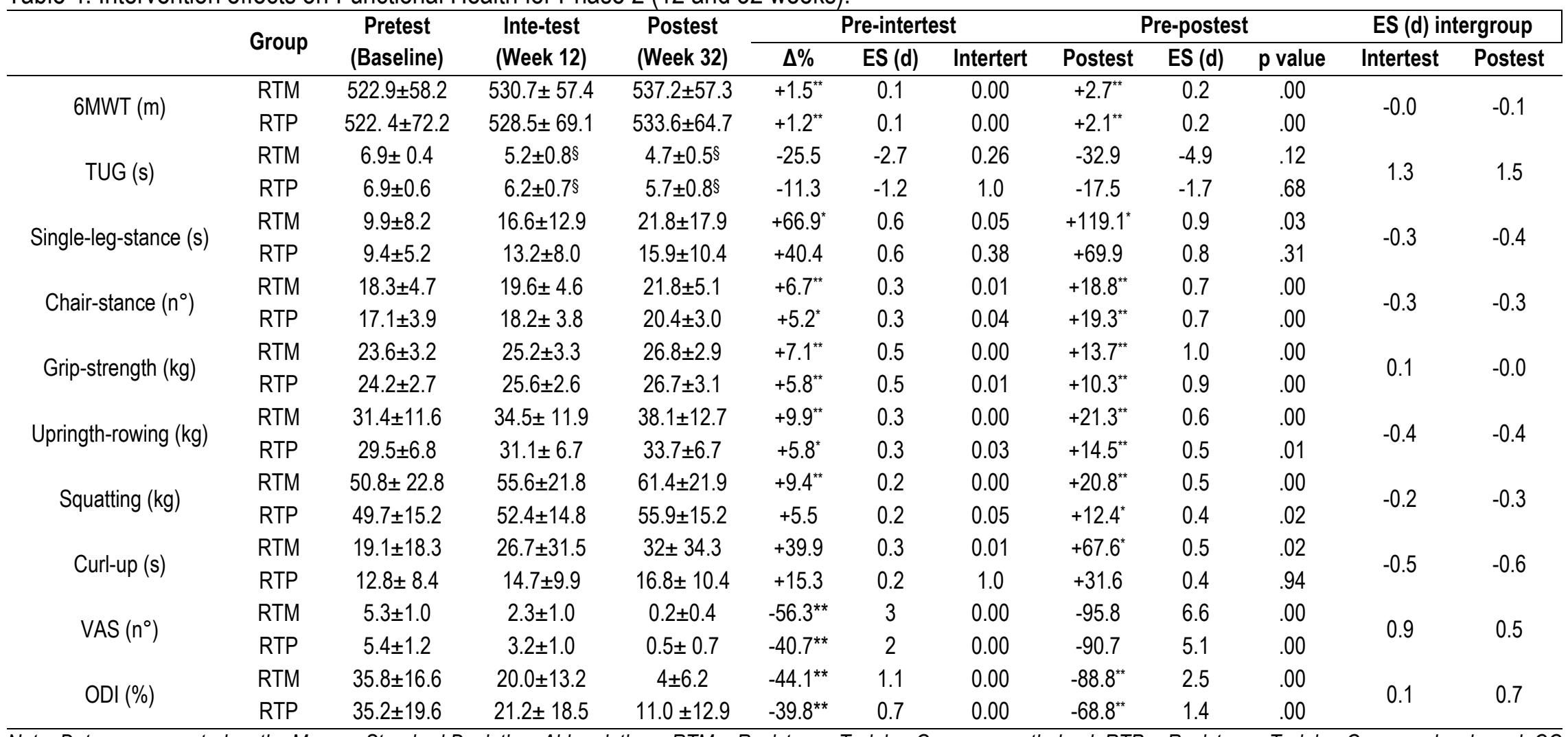

Note. Data are presented as the Mean \pm Standard Deviation. Abbreviations: RTM = Resistance Training Group + menthol gel; RTP = Resistance Training Group + placebo gel; CG = Control Group; $\Delta \%$ = percentage change from pre- to postest; ES: Effect Size; BMI: Body Mass Index; 6MWT: 6minutes walks test; TUG: timed up and go; VAS: Visual Analogue Scale; ODI: Oswestry disability index. ES: $<0.2=$ trivial effect; $0.2-0.5=$ small effect; $0.5-0.8=$ moderate effect and $>0.8=$ large effect; Intragroup differences $\left({ }^{*} p \leq .05\right.$ and ${ }^{* *} p \leq$ .01); $\S$ Intergroup difference $=p \leq .01$ 


\section{DISCUSSION}

The main finding of the present study was that the training program proved to be a lot more effective in both the short and long term when the menthol gel was administered prior to the physical exercise sessions. One of the reasons that may explain this phenomenon is that OAs affected by CLP benefit the analgesic effects of menthol by presenting spinal instability at baseline (Kuo et al., 2015). In turn, this analgesic effect allows them to produce a greater tetanic strength of the musculature (Kuo et al., 2015) and to better reach the prescribed intensity during training sessions. Consequently, this greater and better stimulus could enhance the improvements acquired by the said strength training because the muscles of the lumbar region would be strengthened as a consequence of the stimulus generated by the traction of the elastic material during different exercises. This muscular stress during exercise promotes the contraction of the deep muscles of the trunk, especially transverse abdominal and multifidus (Ferreira et al., 2006), with which the treatment can become more effective (MacDonald et al., 2006). However, it must be taken into account that the analgesic effect of menthol could mask the pain generated by the compensations adopted by OAs during training, with which before starting with the application of the gel, compliance with the stage of prior familiarization with respect to the correct technique of each exercise and the adequate intensity of exercise is absolutely essential. Reason why in this study the familiarization period was highly controlled, and the use of the gel was incorporated until the 4th week of training during the first phase. This is one of the possible reasons why there were no adverse effects or why no dropouts were associated with incorrect practice in any of the training groups, including the group that used the placebo gel.

In the present study, it has been shown that a 12-week-intervention was enough to obtain a clinically significant decrease in the pain at two points evaluated with VAS (Ostelo \& de Vet, 2005) in all the participants of the intervention groups (RTM and RTP), and this effect was maintained after the detraining period until week 32. To date, only two studies have reported a clinically significant decrease in pain after combining the effect of a menthol gel with a non-pharmacological treatment, such as the application of chiropractic adjustments (Zhang et al., 2008) and electrotherapy (Greenstein et al., 2013). However, they were carried out in the adult and young adult population, respectively, and they were invasive and therapist-dependent techniques for physiotherapeutic use. These studies in question are obviously different from the present study because the training protocol used in this study allows more than one patient to be treated with exercise at the same time and the menthol gel was self-applicable by OAs. Likewise, associated with pain reduction, the RTM and RTP groups obtained a clinically significant decrease in their short-term and long-term disability, evaluated with the ODI functional disability questionnaire, and these results are consistent with those reported by Slade \& Keating (2006) and Wang et al. (2012) in their studies conducted with OAs with CLP.

Another of the notable findings in this study was the great effectiveness of moderate-high intensity training with elastic bands in OAs with CLP. In the short and long term, this protocol stimulated improvements in all the parameters that characterized the physical function of the participants, which is directly related to a lower risk of falls, disability, and frailty in OAs (Kim et al. 2010). These benefits produced by the used protocol include a clinically significant improvement in the walking speed (> $30.5 \mathrm{~m}$ ) (Bohannon \& Crouch, 2017), reaching normal parameters in the balance tests, and an increase in strength and muscular endurance. In agreement with previous publications, these findings confirm the improvements in the OA community with no changes in the body weight after short periods of intervention ( 6 to 12 weeks) but with a higher weekly training frequency (3 times a week). For example, the study by Motalebi \& Loke (2014) reported improvements of around $30 \%$ in muscle strength of the lower extremities and dynamic balance evaluated with TUG, after the administration of a training program with elastic tubes at moderate intensity for 12 weeks. While the percentages of improvement obtained by Cavani et al. (2002) are much lower in the 6MWT (+9\%) and in the 
TUG (-15\%), similar results were obtained for the Chair-stance strength test (+30\%) after 6 weeks of training using resistance machines with a protocol of 12 to 15 repetitions until fatigue.

Another point to be highlighted, which may explain the results of this study, is the decrease in pain intensity and lower pain catastrophizing after the intervention program (Coyle et al., 2017; Searle et al., 2015), which reduced the intrinsic limitation in the spinal mobility ranges in OAs with CLP, allowing better performance in functional tests (Coyle et al., 2017; Kuss et al., 2015). This is relevant because, in a previous study, Weiner et al. (2008) used analgesia prior to the training session by means of percutaneous electrical nerve stimulation 5 and 30 min prior to the training program that included flexibility exercises and of aerobic resistance twice a week, for six weeks. Weiner et al. (2008) found that this training program did not enhance the analgesic effects of percutaneous electrical stimulation, nor did it improve the physical function of OAs, although the participants did report a decrease in their pain.

At the same time, moderate-high intensity training has been shown to reverse sarcopenia in OAs, which is accentuated in the presence of CLP (Sakai et al., 2017), which was reflected in the maintenance and/or increase in the percentage of muscle mass directly proportional to the intervention time, enhanced by the menthol gel. On the contrary, the absence of exercise in the CG generated maladaptive changes that caused a worsening in functional performance and body composition, both conditions being related to increased pain in OAs.

Regarding the improvements in body composition parameters, although the literature has shown that longterm improvements in OAs are more significant when selecting a mixed intervention approach, such as diet and exercise (Bouchard et al., 2009; Candow et al., 2011), concern remains regarding the importance of maintaining lean mass after body weight loss, as loss of lean mass as a consequence of dietary restriction can lead to further functional decline (Candow et al., 2011). It is for this reason that the results obtained in this study were compared with the work of Avila et al. (2010), who studied the effect of a dietary intervention that reduced caloric intake by $10 \%$ and its combined effect with a moderate intensity training plan in overweight and obese OAs. After the intervention, it was possible to show that the group that was intervened with a diet obtained a decrease in the body weight by $2 \%$, in fat mass by $0.2 \%$, and in muscle mass by $0.27 \%$. These modifications reported by Avila et al. (2010) in body composition are similar to those generated in phase 1 of our study, with the difference that moderate-high intensity training did not generate a negative impact on lean mass; on the contrary, it increased it by $3.4 \%$. When the results are compared with the combined intervention of diet and moderate-intensity exercise by this same author, it was seen that the participants generated a greater decrease in weight and body fat (3.6\% and $11.2 \%$, respectively) without damaging their lean mass $(+1.3 \%)$ and that these improvements were similar to those achieved in phase 2 of our study (32 weeks) (-3.2\% body weight and $-12.5 \%$ fat mass). However, our intervention generated a greater long-term increase in the percentage of muscle mass $(+3.2 \%)$. Therefore, it is clear that the practice of physical exercise is essential in order to protect lean mass in this population, and although the fat loss was not the primary objective of our study, the selected training intensity could possibly generate a significant decrease in the lipid content of the muscle and the deposits of adipose tissue that explain the decrease in the fat mass of the participants, without negatively impacting their muscle mass and functional capacity.

Few studies have performed strength training in OAs with CLP or in overweight or obese OAs, with only two articles published by the same author to date: Vincent et al. (2014 a) and Vincent et al. (2014 b). Although in these studies only gait strength and speed were evaluated as variables of changes in physical function, their results coincided in showing that global muscle strength training at moderate-high intensities generates improvements in walking speed and muscular strength of the trunk and extremities, with significant results 
after four months of intervention in the strength of the lower extremities, as proven in the group that trained with menthol gel from week 12. However, the intervention protocols used by Vicent et al. had a higher weekly frequency ( 3 times a week), and analytical muscle resistance machines were used for the work of each muscle group. Regarding the pain perception variables, such as catastrophism and self-report of disability, the results are robust and coincide with our findings, which demonstrate a significant decrease in pain in the medium term (Avila et al., 2010).

High adherence has been previously recognized in the literature as a factor that contributes slightly to the improvement of the level of disability of OAs with low back pain. Hicks et al. (2012) studied in adults with CLP (> 50 years) the variables that predicted a better state of pain after applying a training program for 12 months. This study coincides with ours in several aspects, such as frequency, duration, group intervention modality, and high level of supervision, although it differs from our study in work intensity (volume: 20-30 repetitions, without external resistance) and in that it did not include functional physical tests to assess changes. However, Hicks et al. (2012) were able to determine that adherence to the training program (> $75 \%$ attendance) was the main variable that influenced pain reduction in this population. Therefore, in our study, high adherence ( $91.7 \%$ for phase 1 and $89.3 \%$ for phase 2 ) could be considered a positive factor that would add to the effect of the intervention followed.

For all the reasons previously analysed, it can be emphasized that the success of a training program in OAs with CLP seems to be associated with a long-term, highly supervised group work that generates high adherence, does not reproduce painful symptoms, and reaches an intensity of training capable of causing physical and functional changes. In these last two points, the complementary use of a menthol gel could be able to contribute and enhance the effects of an intervention program based on training.

Finally, the present study is not exempt from some limitations, such as the small sample size. However, the intervention time and adherence to the training plan compensate for the small sample size. Therefore, future studies should test the effects of these results in a larger sample. Furthermore, in this study, a loss of CG was generated in phase 2 and only female participants attended, so it is not possible to extrapolate these results to male OAs with CLP.

\section{CONCLUSIONS}

The use of a menthol gel prior to the sessions of a moderate-high intensity strength training program carried out with elastic bands reduces pain and enhances the functional improvements achieved as a result of said training, so its correctly individualized use is recommended as a complementary therapy to an intervention program of these characteristics in female OAs with CLP or in overweight or obese OAs. Finally, the longer the intervention program lasts, the greater the improvements are.

\section{ACKNOWLEDGMENTS}

The authors thank the National Service for Older Adults (SENAMA) Los Ríos and the Human Movement Analysis Laboratory of the Austral University of Chile for permitting the use of their facilities to carry out this study. 


\section{REFERENCES}

Aagaard, P., Suetta, C., Caserotti, P., Magnusson, S. P., \& Kjær, M. (2010). Role of the nervous system in sarcopenia and muscle atrophy with aging: strength training as a countermeasure. Scand. J Med Sci Sports, 20(1), 49-64. https://doi.org/10.1111/j.1600-0838.2009.01084.x

Avila, J. J., Gutierres, J. A., Sheehy, M. E., Lofgren, I. E., \& Delmonico, M. J. (2010). Effect of moderate intensity resistance training during weight loss on body composition and physical performance in overweight older adults. Eur J Appl Physiol, 109 (3), 517-525. https://doi.org/10.1007/s00421-010$\underline{1387-9}$

Bohannon, R. W., \& Crouch, R. (2017). Minimal clinically important difference for change in 6-minute walk test distance of adults with pathology: a systematic review. J Eval Clin Pract, 23(2), 377-381. https://doi.org/10.1111/jep.12629

Boscheinen-Morrin, J., \& Conolly, W. B. (2001). The arthritic hand the hand (pp. 183-201). https://doi.org/10.1016/B978-0-7506-4577-5.50021-8

Bouchard, D. R., Soucy, L., Sénéchal, M., Dionne, I. J., \& Brochu, M. (2009). Impact of resistance training with or without caloric restriction on physical capacity in obese older women. Menopause, 16 (1), 6672. https://doi.org/10.1097/gme.0b013e31817dacf7

Candow, D. G., Chilibeck, P. D., Abeysekara, S., \& Zello, G. A. (2011). Short-term heavy resistance training eliminates age-related deficits in muscle mass and strength in healthy older males. $J$ Strength Cond Res, 25(2), 326-333. https://doi.org/10.1519/JSC.0b013e3181bf43c8

Cavani, V., Mier, C. M., Musto, A. A., \& Tummers, N. (2002). Effects of a 6-week resistance-training program on functional fitness of older adults. J Aging Phys Activ, 10(4), 443-452. https://doi.org/10.1123/japa.10.4.443

Cedraschi, C., Luthy, C., Allaz, A. F., Herrmann, F. R., \& Ludwig, C. (2016). Low back pain and healthrelated quality of life in community-dwelling older adults. Eur Spine J, 25(9), 2822-2832. https://doi.org/10.1007/s00586-016-4483-7

Chen, S. M., Liu, M. F., Cook, J., Bass, S., \& Lo, S. K. (2009). Sedentary lifestyle as a risk factor for low back pain: a systematic review. Int Arch Occup Environ. Health, 82(7), 797-806. https://doi.org/10.1007/s00420-009-0410-0

Colado, J. C., García-Massó, X., Pellicer Catalán, M., Alakhdar, Y., Benavent, J., \& Cabeza Ruiz, R. (2010). A comparison of elastic tubing and isotonic resistance exercises. Int J Sports Med, 31(11), 810-817. https://doi.org/10.1055/s-0030-1262808

Colado, J. C., Pedrosa, F. M., Juesas, A., Gargallo, P., Carrasco, J. J., Flandez, J., Chupel M.U \& Naclerio, F. (2018). Concurrent validation of the OMNI-Resistance Exercise Scale of perceived exertion with elastic bands in the elderly. Exp Gerontol, 103, 11-16. https://doi.org/10.1016/i.exger.2017.12.009

Coyle, P. C., Velasco, T., Sions, J. M., \& Hicks, G. E. (2017). Lumbar mobility and performance-based function: an investigation in older adults with and without chronic low back pain. Pain Med, 18(1), 161-168. https://doi.org/10.1093/pm/pnw136

Edmond, S. L., \& Felson, D. T. (2000). Prevalence of back symptoms in elders. J Rheumatol, 27(1), 220225.

Fairbank, J. C., \& Pynsent, P. B. (2000). The Oswestry disability index. Spine, 25(22), 2940-2953. https://doi.org/10.1097/00007632-200011150-00017

Ferreira, P. H., Ferreira, M.L., Maher,C.G., Herbert, R. D., \& Refshauge, K. (2006). Specific stabilisation exercise for spinal and pelvic pain: a systematic review. Aust J Physiother, 52(2), 79-88. https://doi.org/10.1016/S0004-9514(06)70043-5 
Foster, N. E., Anema, J. R., Cherkin, D., Chou, R., Cohen, S. P., Gross, D. P., ... \& Turner, J. A. (2018). Prevention and treatment of low back pain: evidence, challenges, and promising directions. Lancet, 391(10137):2368-2383. https://doi.org/10.1016/S0140-6736(18)30489-6

Fritz, N. B., Juesas, Á., Gargallo, P., Calatayud, J., Fernández-Garrido, J., Rogers, M. E., \& Colado, J. C. (2018). Positive effects of a short-term intense elastic resistance training program on body composition and physical functioning in overweight older women. Biol Res Nurs, 20 (3), 321-334. https://doi.org/10.1177/1099800418757676

Gargallo, P., Colado, J. C., Juesas, A., Hernando-Espinilla, A., Estañ-Capell, N., Monzó-Beltran, L., ... \& Sáez, G. T. (2018). The Effect of Moderate-Versus High-Intensity Resistance Training on Systemic Redox State and DNA Damage in Healthy Older Women. Biol Res Nurs, 20 (2), 205-217. https://doi.org/10.1177/1099800417753877

Greenstein, J. S., Barton, N. Bishop, P.T., Robert, V. (2013). Evaluation of transcutaneous electrical nerve stimulation vs. Biofreeze in the treatment of back pain. (Abstract). J Chiropr Med, 27(1):90-91.

Hägg, O., Fritzell, P., \& Nordwall, A. (2003). The clinical importance of changes in outcome scores after treatment for chronic low back pain. Eur Spine J, 12(1), 12-20. https://doi.org/10.1007/s00586-0020464-0

Haldeman, S., \& Dagenais, S. (2008). What have we learned about the evidence-informed management of chronic low back pain? Spine J, 8 (1), 266-277. https://doi.org/10.1016/i.spinee.2007.10.026

Hayden, J. A., Van Tulder, M. W., \& Tomlinson, G. (2005). Systematic review: strategies for using exercise therapy to improve outcomes in chronic low back pain. Ann Intern Med, 142(9), 776-785. https://doi.org/10.7326/0003-4819-142-9-200505030-00014

Henchoz, Y.,\& Kai-Lik, So A. (2008). Exercise and nonspecific low back pain: a literature review. Joint Bone Spine, 75(5), 533-9. https://doi.org/10.1016/j.jbspin.2008.03.003

Hicks, G. E., Benvenuti, F., Fiaschi, V., Lombardi, B., Segenni, L., Stuart, M., ... \& Macchi, C. (2012). Adherence to a community-based exercise program is a strong predictor of improved back pain status in older adults: an observational study. Clin J Pain, 28(3), 195. https://doi.org/10.1097/AJP.0b013e318226c411

Hofheinz, M., \& Schusterschitz, C. (2010). Dual task interference in estimating the risk of falls and measuring change: a comparative, psychometric study of four measurements. Clin Rehabil,24 (9), 831-842. https://doi.org/10.1177/0269215510367993

Hubley-Kozey, C. L., McCulloch, T. A., \& McFarland, D. H. (2003). Chronic low back pain: a critical review of specific therapeutic exercise protocols on musculoskeletal and neuromuscular parameters. J Man Manip Ther, 11(2), 78-87. https://doi.org/10.1179/106698103790826419

Ishak, N. A., Zahari, Z., \& Justine, M. (2016). Effectiveness of strengthening exercises for the elderly with low back pain to improve symptoms and functions: A systematic review. Scientifica. https://doi.org/10.1155/2016/3230427

Johar, P., Grover, V., Topp, R., \& Behm, D. G. (2012). A comparison of topical menthol to ice on pain, evoked tetanic and voluntary force during delayed onset muscle soreness. Int J Sports Phys Ther, 7(3), 314.

Katz, R.T. (2006). Impairment and disability rating in low back pain. Clin Occup Environ Med, 5(3), 71940.

Kim, J. W., Eom, G. M., Kim, C. S., Kim, D. H., Lee, J. H., Park, B. K., \& Hong, J. (2010). Sex differences in the postural sway characteristics of young and elderly subjects during quiet natural standing. Geriatr Gerontol Int, 10(2), 191-198.

Kuo, Y. L., Huang, K. Y., Chiang, P. T., Lee, P. Y., \& Tsai, Y. J. (2015). Steadiness of spinal regions during single-leg standing in older adults with and without chronic low back pain. PloS one, 10(5), e0128318. https://doi.org/10.1371/journal.pone.0128318 
Kuss, K., Becker, A., Quint, S., \& Leonhardt, C. (2015). Activating therapy modalities in older individuals with chronic non-specific low back pain: a systematic review. Physiotherapy, 101(4), 310-318. https://doi.org/10.1016/i.physio.2015.04.009

Leboeuf-Yde, C., Fejer, R., Nielsen, J., Kyvik, K. O., \& Hartvigsen, J. (2011). Consequences of spinal pain: do age and gender matter? A Danish cross-sectional population-based study of 34,902 individuals 20-71 years of age. BMC Musculoskelet Disord, 12 (1), 39. https://doi.org/10.1186/14712474-12-39

MacDonald, D. A., Moseley, G. L., \& Hodges, P. W. (2006). The lumbar multifidus: does the evidence support clinical beliefs? Man Ther, 11(4), 254-263. https://doi.org/10.1016/j.math.2006.02.004

Martin, D., Valdez, J., Boren, J., \& Mayersohn, M. (2004). Dermal absorption of camphor, menthol, and methyl salicylate in humans. J Clin Pharmacol, 44 (10), 1151-1157. https://doi.org/10.1177/0091270004268409

Motalebi, S. A., \& Loke, S. C. (2014). Efficacy of progressive resistance tube training in community dwelling older adults: A pilot study. Int $J$ Gerontol, 8 (4), 213-218. https://doi.org/10.1016/j.ijge.2013.12.007

National health and nutrition examination survey (NHANES) (2009). Anthropometry Procedures Manual.United States: Center for Disease Control and Prevention.

Ostelo, R. W., \& de Vet, H. C. (2005). Clinically important outcomes in low back pain. Best Pract Res Cl Rh, 19(4), 593-607. https://doi.org/10.1016/j.berh.2005.03.003

Page, P., \& Alexander, L. (2017). The Clinical Effectiveness of Biofreeze Topical Analgesic on Musculoskeletal Pain: A Systematic Review. J Perform Health, 10122017(1), 1641.

Podichetty, V. K., Mazanec, D. J., \& Biscup, R. S. (2003). Chronic non-malignant musculoskeletal pain in older adults: clinical issues and opioid intervention. Postgrad Med J, 79 (937), 627-633. https://doi.org/10.1136/pmj.79.937.627

Reid, M. C., Williams, C. S., \& Gill, T. M. (2005). Back pain and decline in lower extremity physical function among community-dwelling older persons. J Gerontol A Biol Sc, 60(6), 793-797. https://doi.org/10.1093/gerona/60.6.793

Rikli, R. E., \& Jones, C. J. (2013). Senior fitness test manual. Human Kinetics.

Sakai, Y., Matsui, H., Ito, S., Hida, T., Ito, K., Koshimizu, H., \& Harada, A. (2017). Sarcopenia in elderly patients with chronic low back pain. Osteoporos Sarcopenia, 3(4), 195-200. https://doi.org/10.1016/j.afos.2017.09.001

Searle, A., Spink, M., Ho, A., \& Chuter, V. (2015). Exercise interventions for the treatment of chronic low back pain: a systematic review and meta-analysis of randomised controlled trials. Clin Rehabil, 29(12), 1155-1167. https://doi.org/10.1177/0269215515570379

Slade S. C., Keating J. L. (2006). Trunk-strengthening exercises for chronic low back pain: a systematic review. J Manip Physiol Ther, 29(2):163-173.

Springer, B. A., Marin, R., Cyhan, T., Roberts, H., \& Gill, N. W. Normative values for the unipedal stance test with eyes open and closed (2007). J Geriatr Phys, 30(1), 8-15. https://doi.org/10.1519/00139143200704000-00003

Steib, S., Schoene, D., \& Pfeifer, K. (2010). Dose-response relationship of resistance training in older adults: a meta-analysis. Med Sci Sports Exerc, 42(5), 902-14. https://doi.org/10.1249/MSS.0b013e3181c34465

Sundstrup, E., Jakobsen, M. D., Brandt, M., Jay, K., Colado, J. C., Wang, Y., \& Andersen, L. L. (2014). Acute effect of topical menthol on chronic pain in slaughterhouse workers with carpal tunnel syndrome: triple-blind, randomized placebo-controlled trial. Rehabil Res Pract, 2014:310913. https://doi.org/10.1155/2014/310913 
Topp, R., Brosky, J.A., Pieschel, D. (2013). The effect of either topical menthol or a placebo on functioning and knee pain among patients with knee OA. J Geriatr Phys Ther, 36(2):92-99. https://doi.org/10.1519/JPT.0b013e318268dde1

van der Velde, G., \& Mierau, D. (2000). The effect of exercise on percentile rank aerobic capacity, pain, and self-rated disability in patients with chronic low-back pain: a retrospective chart review. Arch Phys Med Rehab, 81(11), 1457-1463. https://doi.org/10.1053/apmr.2000.9629

van Middelkoop, M., Rubinstein, S. M., Verhagen, A. P., Ostelo, R. W., Koes, B. W., \& van Tulder, M. W. (2010). Exercise therapy for chronic nonspecific low-back pain. Best Pract Res Cl Rh, 24(2), 193204. https://doi.org/10.1016/..berh.2010.01.002

van Tulder, M., Malmivaara, A., Esmail, R., \& Koes, B. (2000). Exercise therapy for low back pain: a systematic review within the framework of the cochrane collaboration back review group. Spine, 25(21), 2784-2796. https://doi.org/10.1097/00007632-200011010-00011

Vincent, H. K., George, S. Z., Seay, A. N., Vincent, K. R., \& Hurley, R. W. (2014a). Resistance exercise, disability, and pain catastrophizing in obese adults with back pain. Med Sci Sports Exerc, 46 (9), 1693. https://doi.org/10.1249/MSS.0000000000000294

Vincent, H. K., Vincent, K. R., Seay, A. N., Conrad, B. P., Hurley, R. W., \& George, S. Z. (2014b). Back strength predicts walking improvement in obese, older adults with chronic low back pain. PM\&R, 6 (5), 418-426. https://doi.org/10.1016/j.pmri.2013.11.002

Vincent HK, Vincent KR, Seay AN, Hurley RW. (2011). Functional Impairment in Obesity: A Focus on Knee and Back Pain. Pain Med,1(5):427-439. https://doi.org/10.2217/pmt.11.39

Wang, X. Q., Zheng, J. J., Yu, Z. W., Bi, X., Lou, S. J., Liu, J., ... \& Shen, H. M. (2012). A meta-analysis of core stability exercise versus general exercise for chronic low back pain. PloS one, 7(12), e52082. https://doi.org/10.1371/journal.pone.0052082

Weiner, D. K., Perera, S., Rudy, T. E., Glick, R. M., Shenoy, S., \& Delitto, A. (2008). Efficacy of percutaneous electrical nerve stimulation and therapeutic exercise for older adults with chronic low back pain: a randomized controlled trial. Pain, 140(2), 344-357. https://doi.org/10.1016/i.pain.2008.09.005

Wright, L. J., Schur, E., Noonan, C., Ahumada, S., Buchwald, D., \& Afari, N. (2010). Chronic pain, overweight, and obesity: findings from a community-based twin registry. J Pain Res, 11 (7), 628-635. https://doi.org/10.1016/i.jpain.2009.10.004

Zhang, J., Enix, D., Snyder, B., Giggey, K., \& Tepe, R. (2008). Effects of Biofreeze and chiropractic adjustments on acute low back pain: a pilot study. J Chiropr Med, 7 (2), 59-65. https://doi.org/10.1016/i.jcme.2008.02.004

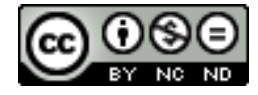

This work is licensed under a Attribution-NonCommercial-NoDerivatives 4.0 International (CC BY-NC-ND 4.0). 